\title{
The coding of odour-intensity in the honeybee antennal lobe: local computation optimizes odour representation
}

\author{
Silke Sachse ${ }^{\dagger}$ and C. Giovanni Galizia \\ Institut für Biologie - Neurobiologie, Freie Universität Berlin, Königin-Luise Str. 28-30, 14195 Berlin, Germany \\ Keywords: Apis mellifera, calcium-sensitive dye, odour processing, olfactory projection neurons, optical recording
}

\begin{abstract}
We investigated strategies involved in odour intensity coding by the primary olfactory centre of insects, the antennal lobe (AL), the structural and functional analogue of the olfactory bulb. Using calcium imaging in the honeybee, we simultaneously measured the projection neuron output responses and a compound signal dominated by receptor neuron input in identified olfactory glomeruli to odours spanning seven log units of concentration. A comparison of the two processing levels indicates that the intercellular computation within the AL modulates and contrast-enhances the primary olfactory signals. As a result the AL network optimizes the olfactory code: odour representation is improved at lower concentrations, the relative activity of olfactory glomeruli allows encoding odour quality over up to four log-unit concentrations, and odour-intensity is reliably represented in the overall excitation across AL.
\end{abstract}

\section{Introduction}

Animals have to recognize odours, which occur in plumes at a variety of concentrations, and both the odour identity and its concentration contain valuable information about the odour source. An important task for the olfactory system is therefore to independently extract both odour quality and its intensity. The first brain area to process olfactory information is the vertebrate olfactory bulb and its insect analogue, the antennal lobe (AL). Both are subdivided into functional processing units, the olfactory glomeruli. The hypothesis that odours are represented by specific spatio-temporal combinatorial patterns of activated glomeruli (Hildebrand \& Shepherd, 1997) has recently been confirmed, among others, by several optical recording studies in vertebrates (Friedrich \& Korsching, 1997; Rubin \& Katz, 1999; Uchida et al., 2000; Fuss \& Korsching, 2001; Meister \& Bonhoeffer, 2001; Wachowiak \& Cohen, 2001; Fried et al., 2002; Spors \& Grinvald, 2002; Wachowiak et al., 2002) and invertebrates (Joerges et al., 1997; Galizia et al., 1999b; Sachse et al., 1999). The afferent input to a particular glomerulus is generally determined by the response profile of a specific olfactory sensory neuron (OSN) type, as each OSN is likely to express only one olfactory receptor and OSNs expressing the same receptor converge onto one or a few glomeruli in vertebrates (Ressler et al., 1994; Vassar et al., 1994; Mombaerts et al., 1996) and in insects (Gao et al., 2000; Vosshall et al., 2000). Local neurons within the bulb or the AL modify the incoming activity, and the result of this computation is relayed to higher order brain centres by the vertebrate mitral cells or the insect projection neurons (PNs).

Yet, the neural mechanisms underlying the processing of odour quality and intensity are poorly understood. Physiological measure-

Correspondence: Dr C. Giovanni Galizia, as above.

E-mail: galizia@zedat.fu-berlin.de

${ }^{\dagger}$ Present address: Rockefeller University, New York, USA.

${ }^{\ddagger}$ Present address: University of California, Riverside 92521, USA.

Received 13 November 2002, revised 19 July 2003, accepted 29 July 2003 ments of OSNs of vertebrates as well as of invertebrates have shown that most OSNs have broadly tuned molecular response profiles (Sicard \& Holley, 1984; Sato et al., 1994; Duchamp-Viret et al., 1999; Malnic et al., 1999), possibly to ensure detection of the vast array of odourant molecules. With increasing odour concentrations these response profiles become broader and less specific (Sato et al., 1994; Malnic et al., 1999), leading to changes of the odour representations reaching the brain. Interglomerular inhibitory interactions have been described in vertebrates and invertebrates (Yokoi et al., 1995; Sachse \& Galizia, 2002). The inhibitory circuits may have evolved in order to sharpen the broader OSN responses and thus to facilitate detection of odour quality. We have addressed this question by simultaneously recording the input and the output of the honeybee $\mathrm{AL}$ while stimulating with odours across a range of concentrations.

In the honeybee 60000 OSNs are distributed on each antenna (Esslen \& Kaissling, 1976) and project to 160 glomeruli of the AL, which can be individually identified based on their shapes and relative positions (Flanagan \& Mercer, 1989; Galizia et al., 1999a). Within the AL the afferent input is processed by up to 4000 local interneurons (LNs) (Witthöft, 1967) and then relayed via three distinct antennocerebralis tracts (ACTs) to higher-order brain centres by 800 projection neurons (PNs) (Hammer, 1997). We used calcium imaging to investigate strategies involved in odour intensity coding in the AL of the honeybee, Apis mellifera. Here, primary alcohols ranging from five carbon atoms (1-pentanol) to ten carbon atoms (1-decanol) elicit activity patterns that are similar to each other with a continuous shift in the maximally activated glomeruli (Sachse et al., 1999). In this study, we selected two direct neighbours of this homologous alcohol series (1-octanol and 1-nonanol) and a more distant substance (1-hexanol), and tested these on a concentration range spanning seven $\log$ units. We combined two different staining protocols in order to simultaneously measure the projection neuron output responses and a compound signal dominated by receptor neuron input (Galizia et al., 1998). By bath-applying the calcium-sensitive dye calcium-green AM to the honeybee brain, all cells in the AL (OSNs, LNs, PNs and glia 
cells) could potentially be stained, as the AM ester allows the dye to permeate cell membranes. However, this signal is not dominated by information from LNs and PNs, as both inhibitory responses and spontaneous activity have never been seen in bath-applied calcium imaging studies (Joerges et al., 1997; Galizia et al., 1998, 1999b; Sachse et al., 1999; this study), but both are prominent in LNs and PNs. This may be due to the numerical dominance of the OSNs. Thus, we take the compound signals as a measure of the functional innervation pattern to the olfactory glomeruli in the AL (i.e. the input). However, with respect to the sensory neuron responses on the antennae, these activities may already be modified as LNs have direct synaptic contacts onto afferent receptor cell terminals. The output of the olfactory glomeruli is accessible by selectively staining PNs with fura-dextran (Sachse \& Galizia, 2002). By combining these two approaches in one animal we can directly measure how the cellular network of the AL shapes the representation of odours. We show that the AL network modulates and optimizes the afferent input by contrast-enhancing the odour representations, improving distinction of odour identity at low concentrations, and increasing concentration-invariant properties of odour-evoked response patterns. As a result, odour intensity is represented in the overall activity, while odour quality is given by the relative activation strength of glomeruli.

\section{Materials and methods}

\section{Preparation and dye loading}

Adult worker honeybees (Apis mellifera) were caught from different hives in the morning. Bees were anaesthetized by cooling, fixed in a Plexiglas stage using dental wax and fed with sucrose solution. A small window was cut into the cuticle on the dorsal side of the head, and glands and tracheae were carefully removed. A glass electrode, which was coated with crystals of the calcium-sensitive dye fura-dextran (potassium salt, $10000 \mathrm{MW}$, Molecular probes, Eugene, OR), dissolved in $2 \%$ bovine serum albumin solution, was inserted into the deutocerebrum lateral to the $\alpha$-lobe, aiming for the projection neurons (PNs) of the lateral antenno-cerebralis tract (1-ACT). We have already shown that this dye application method is specific and effective for fura-dextran (3000 MW) in a previous study (Sachse \& Galizia, 2002). After the dye application the brain was rinsed with Ringer solution (130 mM NaCl, $6 \mathrm{mM} \mathrm{KCl}, 4 \mathrm{mM} \mathrm{MgCl}_{2}, 5 \mathrm{mM} \mathrm{CaCl}_{2}, 160 \mathrm{mM}$ sucrose, $25 \mathrm{~mm}$ glucose, $10 \mathrm{mM}$ HEPES, pH 6.7, $500 \mathrm{mOsmol}$; all chemicals from Sigma) several times to remove extracellular dye. The cut piece of cuticle was repositioned to prevent the brain from drying out. After $3 \mathrm{~h}$ of staining the antennae were immobilized with two silicone components (Kwik-Sil ${ }^{\mathrm{TM}}$, WPI) at their scapus and covered with a coverslip while leaving the brain accessible for a water objective. Afterwards a big window was cut into the cuticle to expose the whole brain, and again remaining glands and tracheae were removed. Only when the PNs were successfully stained with fura-dextran, which was visible by a strong staining of the 1-ACT somata under the fluorescence microscope, did we stain the brain with the second calcium-sensitive dye. Calcium-green 2-AM (50 $\mu \mathrm{g}$; Molecular Probes, Eugene, OR) were first dissolved in $50 \mu \mathrm{L}$ Pluronic in dimethylsuphoxide (DMSO) and then diluted in $950 \mu \mathrm{L}$ Ringer solution. The whole brain was exposed and stained by bath-application. After $1 \mathrm{~h}$ of staining, the brain was washed with Ringer solution in order to remove excess dye and the abdomen was cut.

\section{Odour stimulation}

The animal's antennae were exposed to a constant clean air stream. The odour-laden air was introduced into the continuous air stream to reduce mechanical stimulation. The stimulus timing was computer controlled and lasted for $1 \mathrm{~s}$. Odours used were 1-hexanol, 1-octanol and 1-nonanol (all from Sigma-Aldrich, Deisenhofen). Odourants were dissolved in mineral oil. Dilution was adjusted to equal effective vapour pressure for the different odourants. The control stimulus was a glass cartridge plus filter paper with mineral oil. For each odour, $6 \mu \mathrm{L}$ were placed on filter paper in glass cartridges. Odour concentration was varied by using different dilutions, resulting in different effective amounts on the filter paper (from $10^{-7}$ to $10^{\circ}$ for all odours). Not all odours with all concentration levels were used in all animals. Therefore the number of animals for each stimulus differs. Generally, the experiment started with the presentation of one odour at low concentration followed by higher concentrations of the same odour with the control stimulus in between. The entire stimulus sequence comprising all concentrations of one odour was repeated in each animal.

\section{Imaging}

Imaging was performed using a T.I.L.L. Photonics imaging system (Martinsried, Germany). Monochromatic excitation light alternated between 340, 380 and $475 \mathrm{~nm}$, dicroic: LP $505 \mathrm{~nm}$, emission: LP 515. These filter settings allowed us to measure semisimultaneously the two calcium-sensitive dyes, calcium green 2-AM and fura-dextran. Measurements were made with an upright microscope (Olympus BX 50WI), using a $20 \times$ water-immersion objective (NA 0.5). Pixel image size was $2.4 \times 2.4 \mu \mathrm{m}$, obtained by $2 \times 2$ binning on chip. For each stimulus a series of 60 triple frames was taken with frequencies of $6 \mathrm{~Hz}$. Light was shut off between frames. The stimulus was given at frame 12. The interstimulus interval was $40 \mathrm{~s}$, thus the bee was not exposed to light for approximately $30 \mathrm{~s}$ between measurements.

The number of bees differed depending on the staining procedure. Clear and consistent PN responses were registered in 16 of 65 bees tested. As cell loading with calcium green was only successful on average in 1 of 2 bees, we measured compound signals in only approximately half of the bees (i.e. $n=7$ ), which showed PN responses. Moreover, reliable calcium green signals could only be measured for approximately $30 \mathrm{~min}$, whereas the amplitude of the fura signals remained unchanged for approximately $1.5 \mathrm{~h}$. When using calcium green alone, calcium responses could be measured for approximately $1.5 \mathrm{~h}$ as well (data not shown). Thus, the calcium green dye is probably damaged by the UV light after several measurements. However, during the first $30 \mathrm{~min}$ both dyes could be measured.

\section{Data processing and analysis}

All analyses were carried out using custom software written in IDL (Research Systems, CO). Raw data were median-filtered for shot noise reduction (filter size 3 pixels in two spatial and one temporal dimension). We applied a spatial unsharp-mask filter to each frame in the raw data. Specifically, let A be one raw data image frame, and $\operatorname{sm}(A)$ the same image after a low-pass filter with a kernel of $50 \mu \mathrm{m}$ (corresponding to the size of a glomerulus) has been applied, we then calculated $A_{\text {sharp: }}$ :

$$
A_{\text {sharp }}=2 A-\operatorname{sm}(A)
$$

This algorithm reduces the effect of scattered light produced by strongly activated glomeruli to neighbouring non or less-active glomeruli, which otherwise would appear as false-positive glomeruli. The calcium green signals (i.e. $475 \mathrm{~nm}$ measurements) were calculated as $\Delta F / F$, where the mean of all frames measured was used as $F$. These measurements were corrected for bleaching by fitting a logarithmic function. In the case of fura-recordings, we calculated the ratio $340 \mathrm{~nm} / 380 \mathrm{~nm}$ and multiplied it by 100 . As each glomerulus had an individual background fluorescence, all time traces were shifted to zero shortly before the stimulus onset by subtracting the background 
A
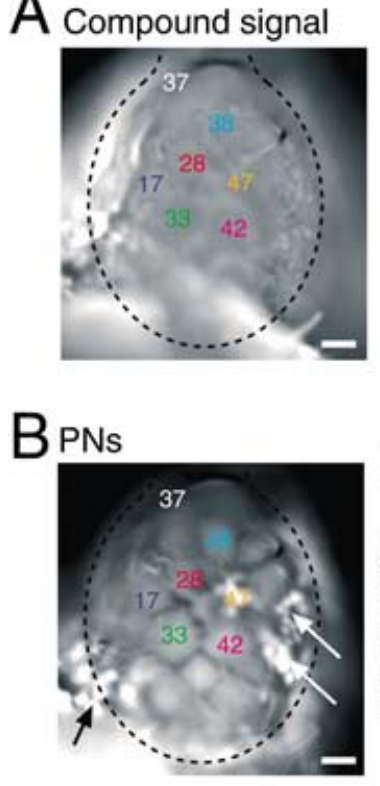

no stimulus

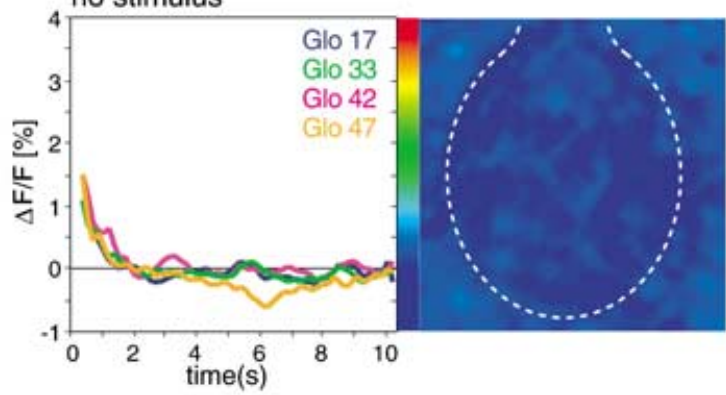

no stimulus

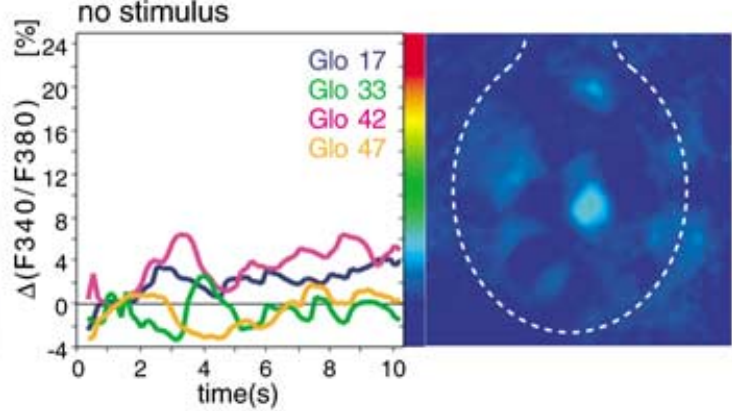

odor application (Mix)

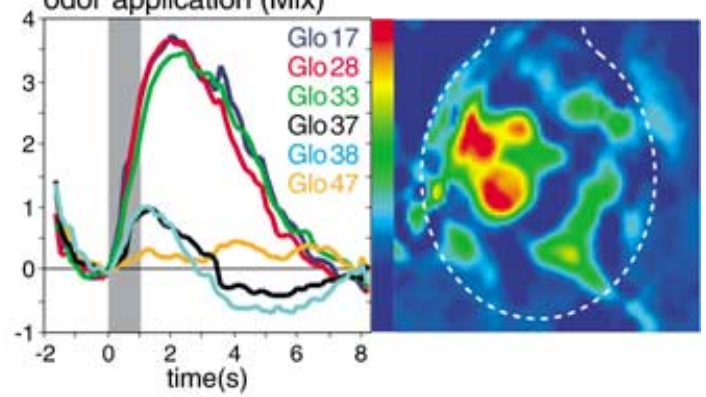

odor application (Mix)

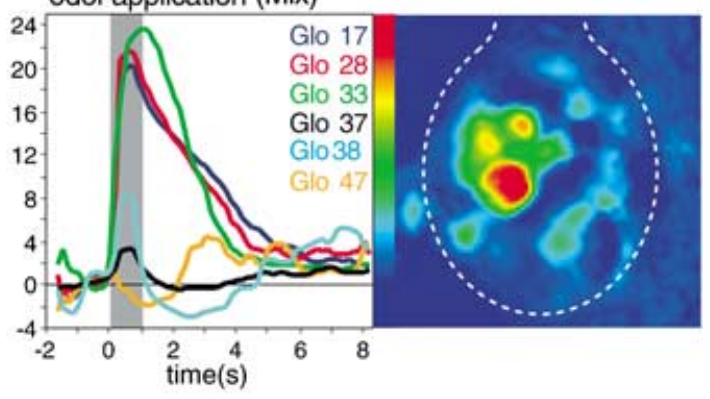

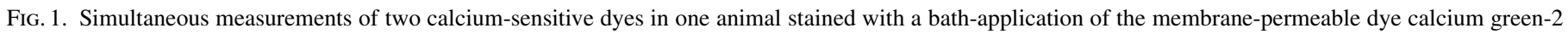

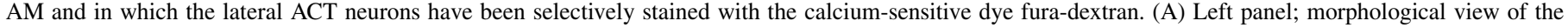

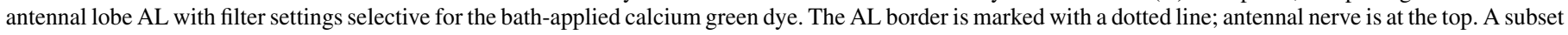

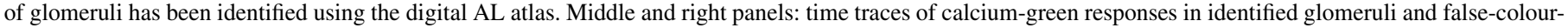

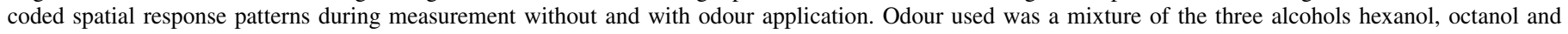

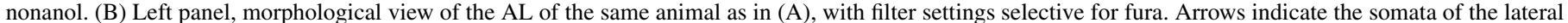

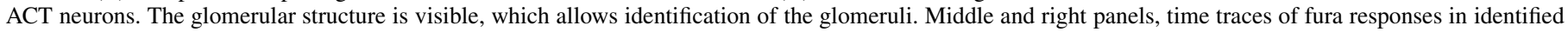

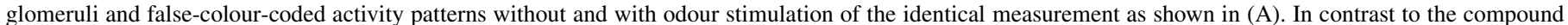

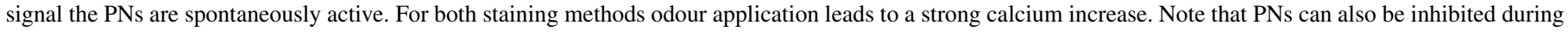
olfactory stimulation (e.g. glomerulus 47). Scale bar, $50 \mu \mathrm{m}$.

using frames 5-9 (i.e. before stimulus); these values are labelled as $\Delta(F 340 / F 380)[\%]$ in the figures. This allows a comparison between the traces of different glomeruli. For false-colour display (Figs 1 and $2 \mathrm{~A}$ ) at each pixel a square of $11 \times 11$ pixels was averaged in its time course; the mean value between frames 12-24 (i.e. from stimulus onset until $1 \mathrm{~s}$ after stimulus offset) is shown for each pixel.

We reconstructed the glomerular borderlines in the fura ratio images (Fig. 1B) and mapped the calcium signals to identified glomeruli using the digital atlas of the honeybee AL (Galizia et al., 1999a). This method has already been used in our previous studies (Galizia et al., 1999b; Sachse et al., 1999; Sachse \& Galizia, 2002). In the honeybee $\mathrm{AL}$, glomeruli are identified by a number and the name of the antennal nerve tract that innervates them, ranging from T1 to T4, e.g. T1-28 or T3-45. All glomeruli in this study were from the T1 tract. We have therefore simplified their names by omitting the T1 affix. Glomerulus 28 in this paper therefore indicates glomerulus T1-28, and accordingly for all other glomeruli. To calculate the numbers of activated and inhibited glomeruli (Figs 2B and 5C) we counted all glomerular calcium increases and decreases greater than noise limit, respectively. Noise was estimated for each animal as the standard deviation of the response between frames 4-11 averaged over all measurements in one animal. All values within the noise limit were clipped to zero. The numbers of activated glomeruli and the dose-response curves between the compound and the PN signals were tested with a standard least squares model fit (ANOVA) using JMP 3.2.1, Statistical Discovery Software (SAS, Cary, NC). The ANOvA was applied to the whole dataset including all concentrations and animals. As not all odours were tested repeatedly in all animals, a repeated-measurements ANOVA was not allowed. Therefore, we averaged repeated measurements of single animals before statistical analysis to avoid unbalanced testing. The resulting single value per animal was used for the ANOVA. Taking the first occurrence of each measurement yielded equally significant results (data not shown). For the signals' amplitude (Fig. 2D) we computed the maximum during frames 12-24 (i.e. from onset until $1 \mathrm{~s}$ after stimulus offset); the signals' duration (Fig. 2E) was calculated at its half-maximal response. Both the calcium signals' amplitude as well as their duration were odour concentration-dependent (Fig. 2C-E). In order to include both parameters we used the integral of the glomerular response to calculate the glomerular activation in each animal (Figs 3, 4 and 6). The calcium green signals (compound responses) had a slower time course than the fura signals (PN responses). We used the integral covering frames 12-30 for the compound responses and frames 12-24 for the PNs (i.e. from stimulus onset until $2 \mathrm{~s}$ or $1 \mathrm{~s}$ after stimulus offset, respectively) as the glomerular activation to include comparable parts of the calcium signals for both dyes. Within each animal we took the mean response of two measurements for each odour and concentration. We calculated the glomerular inhibitory responses by measuring the minimum during stimulus application.

In order to average the glomerular responses over all measured animals, the animal's glomerular activations were normalized in two different ways according to the respective approach. We set the strongest glomerular response in each animal to this particular odour at 1 and scaled the other responses accordingly (Fig. 3) to allow a comparison of the dose-response functions between different glomeruli stimulated by the same odour. A comparison of these normalized 
A Dose of hexanol (log dilution)
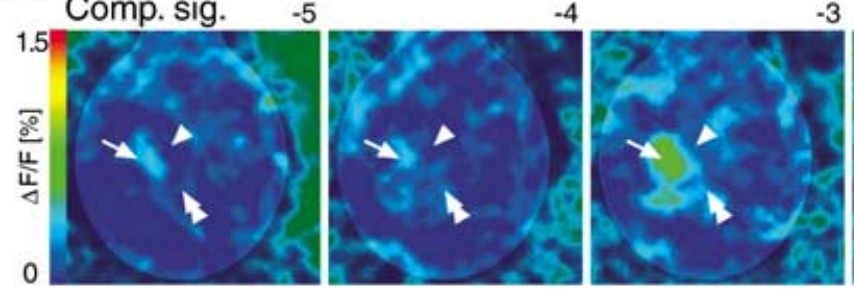

$-3$
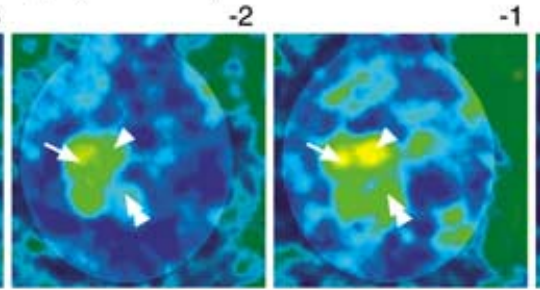

$-1$
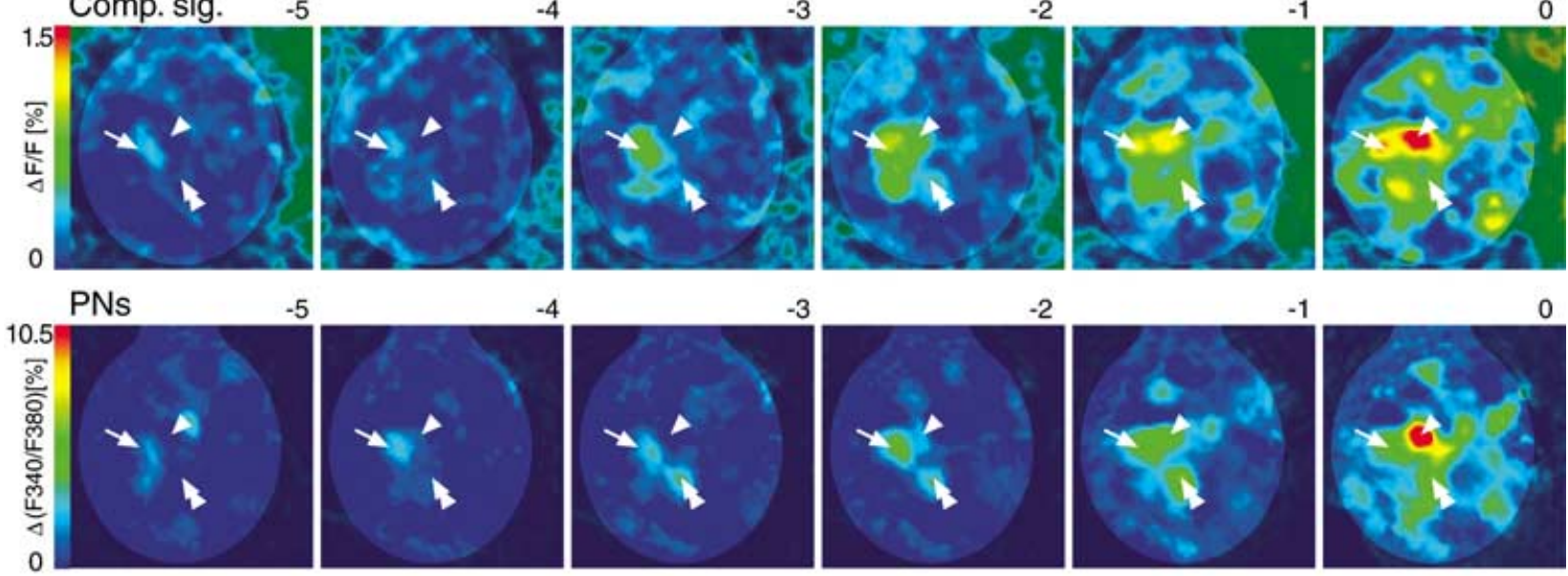

\section{B percent of activated glomeruli}
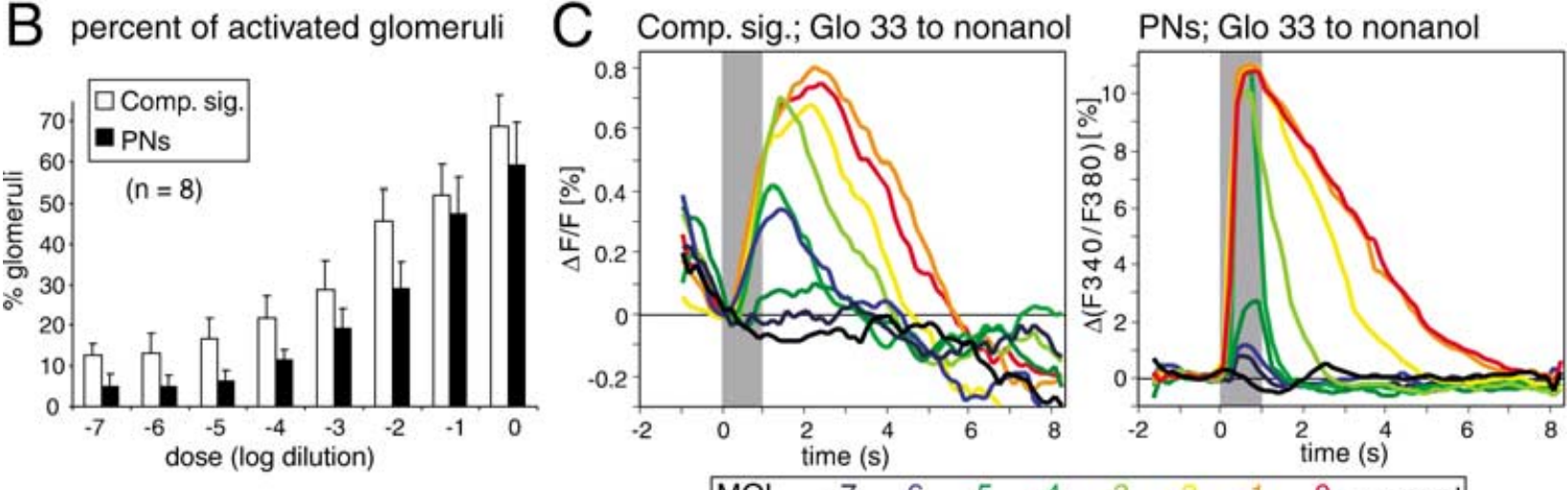

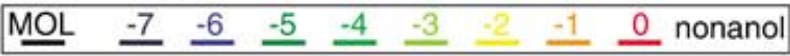
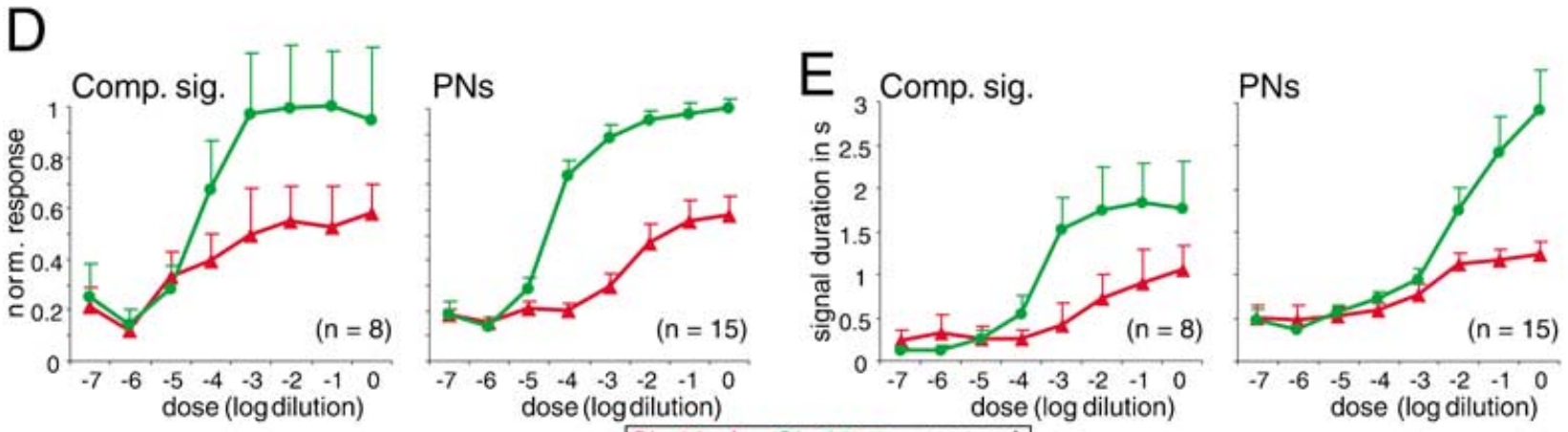

Fig. 2. Increasing odour concentration leads to a recruitment of more glomeruli. (A) False-colour coded spatial activity patterns of the compound signals (above) as well as the PN responses (bottom) to hexanol stimulation over a concentration range of $5 \log$ units. The area outside the AL has been shaded. For comparison the positions of glomeruli 17 (arrow), 28 (arrowhead) and 33 (double arrow) are marked in each image. It is apparent that increasing concentration activates increasing numbers of glomeruli. Note that glomerulus 17 reveals the first detectable signals at low concentration, whereas glomerulus 28 dominates the pattern at the highest concentration. (B) Percent of activated glomeruli of the compound signal (white bars) as well as the PN response (black bars) averaged over all odours and identical animals for both staining procedures; error bars (SEM) and $n$ relate to animals. Both show a strong increase of active glomeruli with increasing odour concentration. Note that the compound signals comprise a significant higher number of activated glomeruli than the PNs at each concentration level $(P<0.0001$, ANOVA). $(C)$ Time traces of glomerulus 33 to nonanol stimulation. Stimulus is given by the grey shading. The odour concentration is coded by the different colours. For both the compound signal as well as the PN response increasing nonanol concentration leads to an increase in the signal amplitude and duration until saturation. (D and E) Plots of signal amplitudes (D) and duration (E) vs. concentration for glomeruli 28 and 33 to different nonanol concentrations averaged over all individuals. Error bars indicate means and SE values across animals. Strongly, as well as intermediately activated glomeruli show an approximately sigmoidal curve. Note that the number of bees differs between staining procedures (see Materials and methods).

response intensities between dose-response functions to different odours is not meaningful, as they are scaled to different values. Therefore in a parallel analysis, we set the strongest glomerular activation across all measured odours and glomeruli in each animal at 1 and scaled all other responses accordingly (Fig. 4A and B). This allows comparing the dose-response function to different odours within one glomerulus. For example, the compound response of glomerulus 28 to hexanol showed the maximal activation for the 

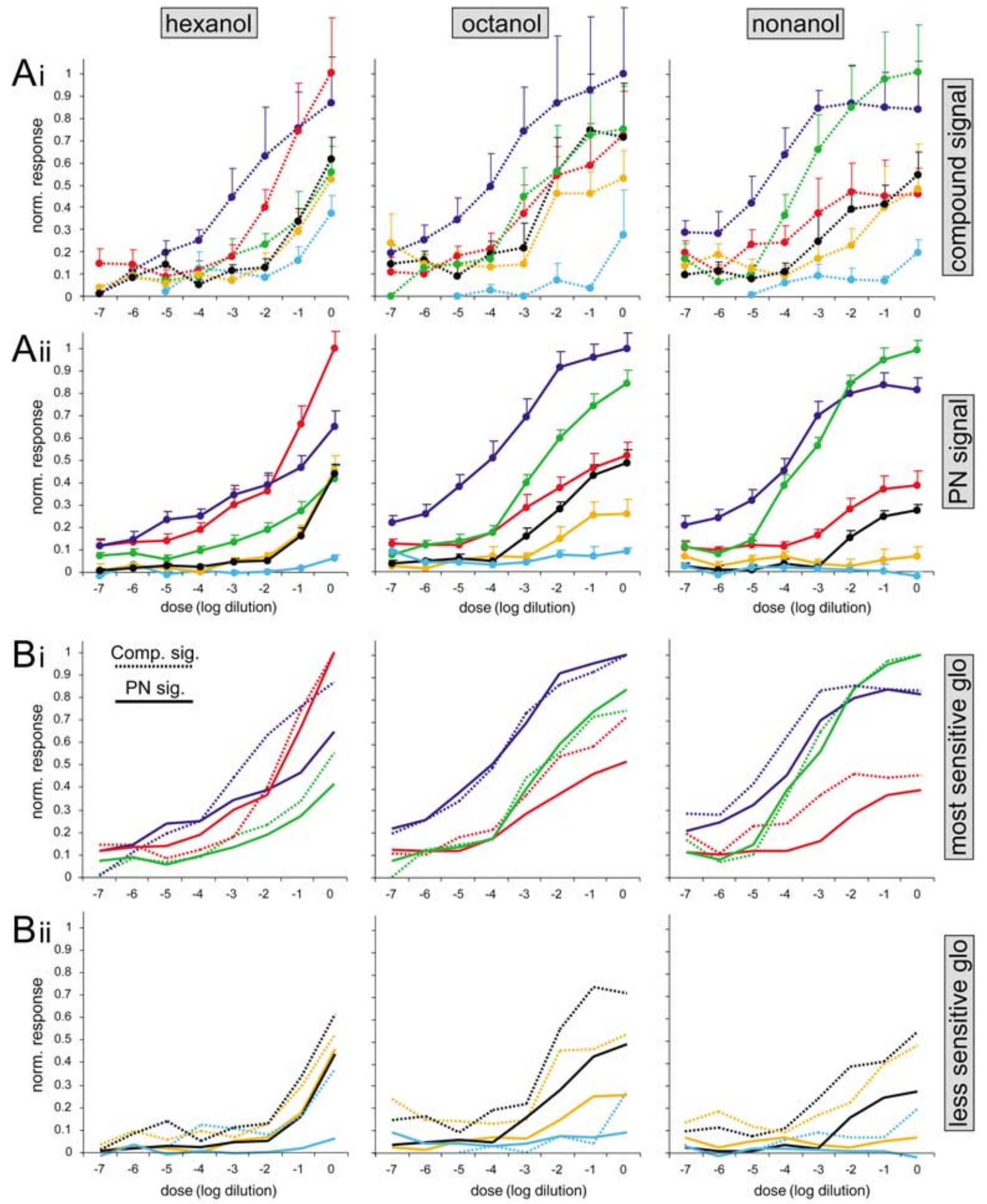

Glo $17(n=7-15) ;$ Glo $28(n=7-15) ;$ Glo $33(n=7-15) ;$ Glo $38(n=7-15) ;$ Glo $39(n=2-6) ; \underset{\text { Glo }}{ } 52(n=7-15)$

FIG. 3. Dose-response functions of different identified glomeruli to a specific odour. (A) Compound responses (dotted lines, Ai) and PN responses (solid lines, Aii) of six identified glomeruli to the three primary alcohols hexanol, octanol and nonanol to eight concentrations averaged over all animals measured (means and SEM). The responses have been normalized by setting the strongest glomerular response in each animal for each odour at 1 and scaling the other responses accordingly to eliminate individual differences in response intensity. Thus, a comparison between the dose-response functions of different odours is not meaningful. Most of the dose-response curves are sigmoidal, but some curves do not saturate. (B) For comparison between the compound and the PN dose-response functions, the same curves as in (A) were superimposed, separated in most sensitive (Bi) and less sensitive glomeruli (Bii). In all cases the dose-response curves of the compound signal are either identical or above the curves of PNs, indicating that the signals on the PN level are more confined compared to the compound signal. 

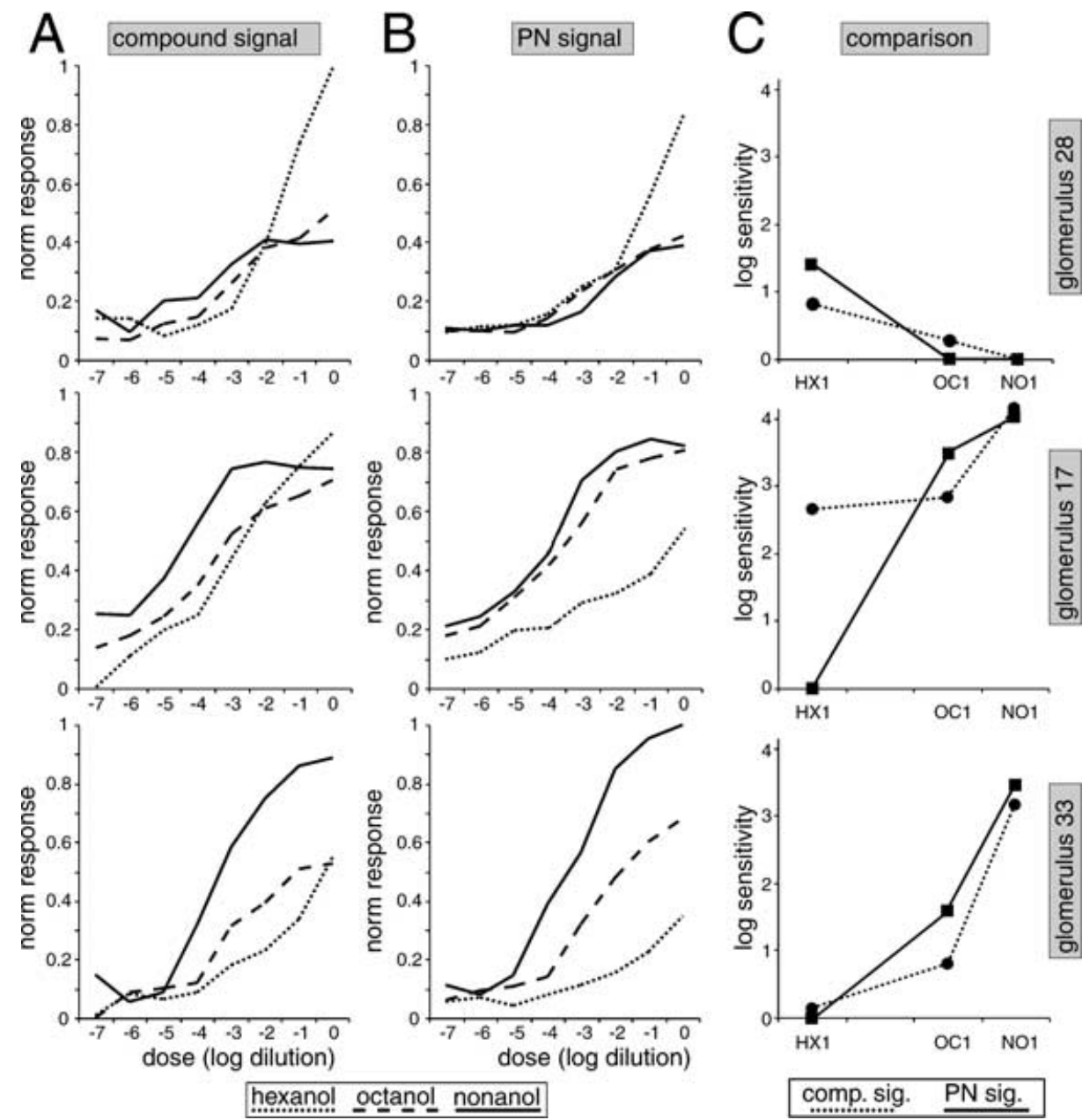

Fig. 4. Dose-response functions and sensitivities of identified glomeruli to different odours. (A and B) For each of the three identified glomeruli 28,17 and 33 their dose-response curves to all three alcohols are represented in one plot for the compound signal (A) as well as for the PN response (B). The curves are normalized to the strongest glomerular response in all three odours. Hexanol elicited the strongest response in glomerulus 28, whereas glomeruli 17 and 33 are most responsive to nonanol. Note that each odour elicits at least an intermediate compound response in each glomerulus, whereas some of these responses are reduced and thus confined on the PN level. Same data as in Fig. 3, but with different normalization. (C) Sensitivities (defined as the inverse of the half-saturating concentration $K_{i}$ ) of the same three glomeruli as in (A) and (B) to the three tested alcohols hexanol (HX1), octanol (OC1) and nonanol (NO1) of the compound and PN signals. The data are represented logarithmically. The arrangement of the three odours reflects their chemical similarity. The sensitivity differs in an odour-specific manner for the compound and PN responses.

highest concentration (Fig. 4A), whereas the PN response was strongest in glomerulus 33 to nonanol (Fig. 4B). We fitted each doseresponse function to the Hill equation using a least-square optimization (see Results). From this function we calculated the half-saturating concentration $\left(K_{i}\right)$, the sensitivity $\left(1 / K_{i}\right)$ and the Hill coefficient as a measure of the slope $(n)$ for a subset of identified glomeruli to each tested odour (Table 1).

Each odour representation can be regarded as a vector in a multidimensional space, where each glomerulus represents one dimension. Because it is not possible to visualize points in a 23-dimensional space, we used a principal component analysis (PCA). This technique calculates a new cartesian coordinate system based on the eigenvectors of the correlation matrix in which the first axis (i.e. the first principal component, PC1) explains most of the variance observed, the second (PC2) most of the remaining variance, etc. It therefore allows the visualization of the relative positions of the points observed (i.e. the odour representations defined by the glomerular responses) in a reference system with reduced dimensionality (Fig. 6B). We included a varimax rotation of the first two principal components to make the factors more interpretable. The varimax method tries to shift elements of this matrix towards 1 or 0 to show the clustering of variables. The PCA was performed using SPSS (SPSS Inc., Chicago, IL).

\section{Results}

\section{Simultaneous measurements of different $A L$ neuron populations}

Loading two different calcium-sensitive dyes in different cell populations in one animal it is possible to visualize their calcium response properties and thus to simultaneously measure different processing levels. We bath-applied the membrane-permeable calcium green-AM to measure the OSNs, as the resulting compound signal is dominated by the input to the AL (Fig. 1A; see Introduction and Galizia et al., 1998). Without any stimulation, the glomeruli revealed no calcium changes above noise level, i.e. no spontaneous activity. Odour application of $1 \mathrm{~s}$ led to a strong, reliable and long-lasting calcium increase in several glomeruli. The time courses showed an exponential decrease during the measurements due to the bleaching of the calcium green dye, which was corrected by subtracting a logarithmic curve fitting the 
TABLE 1. Strongly activated glomeruli display an increased sensitivity on the PN level compared to the compound responses, whereas the sensitivity of loweractivated glomeruli is reduced

\begin{tabular}{|c|c|c|c|c|c|c|}
\hline Glomerulus & Odour & $K_{\mathrm{i}}$-value & Sensitivity & Hill coefficient & Signal & $n$ \\
\hline 17 & $\begin{array}{l}\text { HX1 } \\
\text { OC1 } \\
\text { NO1 }\end{array}$ & $\begin{array}{l}2.2 \times 10^{-3} \\
9.4 \times 10^{-1} \\
1.5 \times 10^{-3} \\
3.2 \times 10^{-4} \\
7.0 \times 10^{-5} \\
9.7 \times 10^{-5}\end{array}$ & $\begin{array}{l}454.55 \\
1.06 \\
666.67 \\
3125.0 \\
14285.7 \\
10309.3\end{array}$ & $\begin{array}{l}0.30 \\
0.14 \\
0.25 \\
0.22 \\
0.24 \\
0.24\end{array}$ & $\begin{array}{l}\text { C. } \\
\text { PN } \\
\text { C. } \\
\text { PN } \\
\text { C. } \\
\text { PN }\end{array}$ & $\begin{array}{r}7 \\
13 \\
7 \\
74 \\
8 \\
8 \\
15\end{array}$ \\
\hline 28 & $\begin{array}{l}\text { HX1 } \\
\text { OC1 } \\
\text { NO1 }\end{array}$ & $\begin{array}{l}1.5 \times 10^{-1} \\
3.8 \times 10^{-2} \\
5.3 \times 10^{-1} \\
\left(5.2 \times 10^{0}\right) \\
\left(6.0 \times 10^{0}\right) \\
\left(1.4 \times 10^{1}\right)\end{array}$ & $\begin{array}{l}6.67 \\
26.32 \\
1.89 \\
(0.19) \\
(0.17) \\
(0.07)\end{array}$ & $\begin{array}{l}0.56 \\
0.31 \\
0.18 \\
(0.15) \\
(0.10) \\
(0.14)\end{array}$ & $\begin{array}{l}\text { C. } \\
\text { PN } \\
\text { C. } \\
\text { PN } \\
\text { C. } \\
\text { PN }\end{array}$ & $\begin{array}{r}7 \\
13 \\
7 \\
74 \\
14 \\
8 \\
15\end{array}$ \\
\hline 33 & $\begin{array}{l}\text { HX1 } \\
\text { OC1 } \\
\text { NO1 }\end{array}$ & $\begin{array}{l}7.4 \times 10^{-1} \\
\left(4.9 \times 10^{1}\right) \\
1.6 \times 10^{-1} \\
2.5 \times 10^{-2} \\
6.8 \times 10^{-4} \\
3.5 \times 10^{-4}\end{array}$ & $\begin{array}{l}1.35 \\
(0.02) \\
6.25 \\
40.0 \\
1470.59 \\
2857.14\end{array}$ & $\begin{array}{l}0.25 \\
(0.19) \\
0.21 \\
0.25 \\
0.39 \\
0.45\end{array}$ & $\begin{array}{l}\text { C. } \\
\text { PN } \\
\text { C. } \\
\text { PN } \\
\text { C. } \\
\text { PN }\end{array}$ & $\begin{array}{r}7 \\
13 \\
7 \\
74 \\
14 \\
8 \\
15\end{array}$ \\
\hline 38 & $\begin{array}{l}\text { HX1 } \\
\text { OC1 } \\
\text { NO1 }\end{array}$ & $\begin{array}{l}1.0 \times 10^{0} \\
\left(2.6 \times 10^{0}\right) \\
\left(4.6 \times 10^{0}\right) \\
\left(7.1 \times 10^{2}\right) \\
\left(1.5 \times 10^{1}\right) \\
-\end{array}$ & $\begin{array}{l}1.0 \\
(0.38) \\
(0.22) \\
(0.001) \\
(0.07) \\
-\end{array}$ & $\begin{array}{l}0.35 \\
(0.51) \\
(0.20) \\
(0.18) \\
(0.15) \\
-\end{array}$ & $\begin{array}{l}\text { C. } \\
\text { PN } \\
\text { C. } \\
\text { PN } \\
\text { C. } \\
\text { PN }\end{array}$ & $\begin{array}{r}7 \\
13 \\
7 \\
74 \\
14 \\
8 \\
15\end{array}$ \\
\hline 52 & $\begin{array}{l}\text { HX1 } \\
\text { OC1 }\end{array}$ & $\begin{array}{l}4.5 \times 10^{-1} \\
\left(3.0 \times 10^{0}\right) \\
2.8 \times 10^{-1} \\
\left(3.7 \times 10^{0}\right) \\
\left(1.4 \times 10^{0}\right) \\
\left(2.8 \times 10^{1}\right)\end{array}$ & $\begin{array}{l}2.22 \\
(0.34) \\
3.57 \\
(0.27) \\
(0.71) \\
(0.04)\end{array}$ & $\begin{array}{l}0.40 \\
(0.53) \\
0.21 \\
(0.23) \\
(0.18) \\
(0.24)\end{array}$ & $\begin{array}{l}\text { C. } \\
\text { PN } \\
\text { C. } \\
\text { PN } \\
\text { C. } \\
\text { PN }\end{array}$ & $\begin{array}{r}7 \\
12 \\
7 \\
13 \\
8 \\
14\end{array}$ \\
\hline
\end{tabular}

Summary of $K_{\mathrm{i}}$-values, sensitivities (i.e. $1 / K_{\mathrm{i}}$ ) and Hill coefficients obtained for the five most highly activated glomeruli tested with all three odours over a concentration range of seven log units. These parameters are listed for the compound (C.) and the PN response (PN); numbers of animals are given in the last column (n). Parameters were calculated by fitting the dose-response functions to the Hill equation (Eqn 1). The dose-response functions were normalized as described in Fig. 4A and B. The values of dose-response functions that did not achieve their half-saturating concentration were estimated and are therefore shown in brackets. Empty entries indicate responses below noise limits, i.e. no dose-response function could be fitted.

bleaching decrease (see Materials and methods). As this correction could not completely eliminate the bleaching, this artefact is still visible in the time courses of the compound measurements.

With the second and membrane-impermeable calcium-sensitive dye, fura-dextran, we selectively backfilled and measured the PNs of the lateral ACT, which represent the output of the superficial glomeruli (Sachse \& Galizia, 2002). Loading of the PNs was apparent when the somata of the lateral ACT neurons were stained (arrows in Fig. 1B) and when the glomerular structure was visible in the fluorescence ratio image. This allowed us to reconstruct the glomerular borderlines and map the calcium signals to morphologically identified glomeruli. In contrast to the compound signals, PNs revealed a high amount of spontaneous activity above noise level in the absence of any odour (compare Fig. 1A and B). Odour stimulation evoked both excitatory (e.g. glomerulus 17, 28 and 33 in Fig. 1B) as well as inhibitory responses and off-responses (e.g. glomerulus 47 in Fig. 1B) in PNs. Surprisingly, the PN responses had a faster rising time and faster decay time than the compound signals. On average, the compound signals reached their half-maximum value $1200 \mathrm{~ms}$ after stimulus onset, whereas the PN responses took only $200 \mathrm{~ms}$. In control experiments using calcium green dextran to label the PNs, we observed the same response dynamics as compared to the fura stainings (data not shown). Thus the observed differences must be related either to the use of $\mathrm{AM}$ or to the differences in the calcium influx and buffering mechanisms of the measured cells and of presynaptic vs. postsynaptic calcium influx. Other calcium imaging studies, where the afferents were selectively labelled with calcium green dextran, also showed prolonged calcium responses to odours as our calcium green AM signals (Friedrich \& Korsching, 1997; Wachowiak \& Cohen, 2001; Wachowiak et al., 2002) and also long rise times (Friedrich \& Korsching, 1997).

Neither spontaneous activity nor inhibitory responses were seen in the compound signals, confirming that we measured both dyes independently from each other. Relative excitation of the activated glomeruli showed slight differences for the compound signals and the PNs. In the example shown in Fig. 1, glomeruli 17, 28 and 33 were activated similarly in the compound signal, whereas the $\mathrm{PN}$ response was stronger for glomerulus 33 than for glomeruli 17 and 28, indicating that the AL network modulates odour-evoked activity patterns.

\section{Spatial and temporal activity patterns are concentration-dependent}

We measured spatio-temporal dose-response curves to the three odours 1-hexanol, 1-octanol and 1-nonanol diluted in seven log units in the solvent mineral oil. Increasing odour concentration led to an increased number of activated glomeruli (Fig. 2A and B). For example, the odour hexanol (Fig. 2A) elicited the first detectable signals in glomerulus 17 (white arrow in Fig. 2A). With increasing odour 
concentration glomeruli 33 (double arrowhead) and 28 (arrowhead) also became activated. The latter was finally the dominant glomerulus for the highest odour concentration. The concentration-dependent recruitment of activated glomeruli was seen in all preparations for all odours tested resulting in activity patterns that differ for different odour concentrations. These findings are consistent with several optical recording studies of the olfactory bulb of rats, mice and zebrafish (Friedrich \& Korsching, 1997; Rubin \& Katz, 1999; Johnson \& Leon, 2000; Fuss \& Korsching, 2001; Meister \& Bonhoeffer, 2001; Wachowiak \& Cohen, 2001) as well as studies of the honeybee and moth AL (Sachse et al., 1999; Galizia et al., 2000). However, imaging of OSNs in turtles showed that odourants at even low concentrations elicited activity in a large fraction of the measured glomeruli (Wachowiak et al., 2002).

Interestingly, the compound signals comprised a significant higher number of activated glomeruli (69\% of the imaged glomeruli for the highest concentration; Fig. 2B) than the PN activity patterns (59\% of the imaged glomeruli). In order to quantify this observation statistically, we applied a general model fit ANOVA to the whole dataset in Fig. 2B. The different concentrations ( $F$-ratio 228, $P<0.0001$, $D F=1)$ and compound vs. PN signal $(F$-ratio $=16, P<0.0001$, $D F=1)$ were highly significant, the difference between individual animals only to $2 \%$ ( $F$-ratio $2.5, P=0.018, D F=7$ ).

The responses increased in amplitude and duration with increasing odour concentration until a saturated maximum. An example for the time courses of an identified glomerulus during stimulation with nonanol at increasing concentrations (marked by the different colours) is shown in Fig. 2C. The compound signals and the PN responses showed a similar concentration dependence; both revealed a successive increase and prolongation of the calcium responses following the increasing concentration. The maximal amplitude and signal duration of two identified glomeruli during nonanol stimulation averaged over all animals is shown in Fig. 2D and E. Both parameters showed a saturation level at a specific concentration even for only intermediately activated glomeruli (e.g. glomerulus 28, red line).

\section{Dose-response functions of the compound signal and the output neurons}

As both the signal amplitude as well as the signal duration were concentration-dependent (Fig. 2D and E), we defined the integral covering frames 12-24 for the PNs and frames 12-30 for the compound responses (i.e. from stimulus onset until $1 \mathrm{~s}$ or $2 \mathrm{~s}$ after stimulus offset) as the glomerular activation. We calculated the dose-response functions for the tested odours by averaging across all measured animals. In total the dose-response curves were measured for 32 glomeruli, tested at eight concentrations over a range of seven log units. Each odour evoked specific dose-response functions in these identified glomeruli. Most of the dose-response curves are sigmoidal with their rising phase spanning up to four log units. The dose-response functions of six exemplary glomeruli are shown in Fig. 3. Note that the curves are normalized to the maximum glomerular response within each odour. Thus, a comparison of the response intensities of specific glomeruli between the different odours is not possible in this figure. In the compound signals (Fig. 3, Ai), hexanol elicited the first responses in glomerulus 17 (blue curve) at a concentration of $10^{-4}$. As glomerulus 28 revealed a steeper slope (red curve), its response ended above that of glomerulus 17 at the highest concentration. The dose-response functions of the other glomeruli were shifted to higher concentrations. For hexanol the glomerular responses did not saturate, possibly because of insufficient receptor activation. Octanol response was clearly dominated by glomerulus 17 , which was already activated above noise threshold at a concentration of $10^{-6}$. Responses to nonanol were also first detectable in glomerulus 17 . However, at a concentration of $10^{-2}$ glomerulus 33 (green curve) took over and dominated the activity pattern. The dose-response functions of the PNs showed similar properties (Fig. 3, Aii); most of the curves were sigmoidal with a dynamic range also spanning up to four log units as for the compound curves. In order to simplify a comparison between the compound signal and the PNs, we replicated the dose-response functions shown in Fig. 3A and superimposed them for three glomeruli each in Fig. 3, Bi and Bii. The dose-response functions of the most sensitive glomeruli (e.g. glomerulus 28 for hexanol, glomerulus 17 for octanol and glomerulus 33 for nonanol) are almost identical for both neuron populations when normalizing for each odour. However, less sensitive glomeruli reveal strong differences between the compound signals and the PNs. For example, the dose-response functions of glomerulus 17 for hexanol, glomerulus 28 for octanol and glomeruli 17 and 28 for nonanol were all shifted to a higher concentration and thus were less activated in the PN response. Glomerulus 39 showed even no $\mathrm{PN}$ response to nonanol, although this glomerulus was activated in the compound pattern.

In Fig. 4A and $\mathrm{B}$ these curves were normalized to the maximum glomerular response including all odours. Glomerulus 28 was most sensitive to hexanol, whereas octanol and nonanol elicited similarly weak responses. Glomeruli 17 and 33 showed their strongest responses to nonanol, intermediate and weak responses to octanol and hexanol, respectively. The last two glomeruli are examples for glomeruli whose response strength is dependent on the carbon chain length of the odour molecule (Rubin \& Katz, 1999; Sachse et al., 1999; Uchida et al., 2000; Meister \& Bonhoeffer, 2001). Again, comparison of the doseresponse curves of both neuron populations reveals that some are rightshifted in their output, while others were not. As this effect differed between odours and glomeruli, we separated glomeruli with strong responses to the odours tested (group 1: glomeruli 28, 17 and 33) and glomeruli with weak responses (group 2: glomeruli 38, 39, 52). Normalized responses were significantly higher in PNs compared to the compound signals in glomeruli of group 1 (mean PN-signal 0.30, mean compound signal 0.20 , difference significant to $P<0.0001$, $F$-ratio $=71, D F=1$, least-squares ANOVA on 1318 observations, correcting for glomerulus $[D F=2]$, odour $[D F=2]$, animal $[D F=15]$ and concentration [continuous variable, $D F=1]$ ), and were significantly lower in PNs compared to compound signals in group 2 (mean PN-Signal 0.07, mean compound signal 0.11, difference significant to $P<0.0001, F$-ratio $=22, D F=1$, least-squares ANOVA on 1017 observations, correcting for glomerulus, odour, animal and concentration, $D F$ s as above).

The dose-response functions can be described quantitatively by fitting the Hill equation to the data:

$$
R=R_{\max }\left[C^{n} /\left(C^{n}+K_{i}^{n}\right)\right]
$$

In this equation $R$ is the measured response amplitude, $R_{\max }$ is the maximal response, $C$ is the odour concentration, $n$ (Hill coefficient) is a measure of the slope and $K_{i}$ is the half-saturating concentration. For a given glomerulus the response strength to a specific odour is determined by its parameter $K_{i}$. We therefore defined the sensitivity of a particular glomerulus to a specific odour as the inverse of $K_{i}$ (definition after Meister \& Bonhoeffer, 2001). The sensitivity of the three prominent glomeruli for the compound signals and for the PNs to the tested odours is graphically shown in Fig. 4C. Table 1 summarizes all parameters, as the $K_{i}$-values, the sensitivities and the Hill coefficients of a subset of glomeruli during measurements of the compound signals $(\mathrm{C})$ and the PN responses $(\mathrm{PN})$, respectively. Consideration of the most strongly activated and prominent glomeruli for each of the three odours (glomerulus 28 for hexanol, 17 for octanol and 33 for 
octanol and nonanol) shows that these have lower $K_{i}$-values and thus a higher sensitivity on the PN level than for the compound response (Fig. 4C, Table.1). For example glomerulus 17 has its half maximum response to octanol at a concentration of $10^{-3}$ in the compound signal compared to $10^{-4}$ on the PN level. In contrast, weaker glomeruli showed higher $K_{i}$-values and lower sensitivities on the PN level as compared to the compound signals. The fitted slopes revealed various Hill coefficients from 0.14 to 0.56 , indicating that different glomeruli can have different dose-response functions. Remarkably, the strongly activated glomeruli have higher Hill coefficients (i.e. steeper slopes) than the weaker-activated glomeruli. For example, the PN doseresponse function of glomerulus 33 had a Hill coefficient of 0.45 to nonanol, whereas glomeruli 17 and 28 had values of only 0.24 and 0.14 , respectively (Table 1 ). The slope and thus the dynamic range of an identified glomerulus to a specific odour was quite similar for the compound response and its output. This is different from vertebrates, where electrophysiological recordings showed a difference in the intensity tuning range for OSNs compared to bulb neurons: singlecell-recordings of individual OSNs have a dynamic range typically spanning only 1-2 log units of concentration (Firestein et al., 1993; Trotier, 1994; Duchamp-Viret et al., 2000) and thus have Hill coefficients from 1.9 to 4.4 (Firestein et al., 1993), as opposed to a range of 2-4 log units in mitral cells (Duchamp-Viret et al., 1990a). As the latter value matches our measured dynamic range of PNs, the discrepancy lies in the OSN data. Indeed, electrophysiological recordings of honeybee OSNs showed narrow tuning ranges, as are found in vertebrates (Vareschi, 1971). The larger dynamic range measured in this study is therefore probably due to the fact that we measured averaged signals of all OSNs converging onto single glomeruli. In consequence, individual receptor capacities with their narrow intensity tuning curves may add up to an overall response with a much broader dynamic range (Cleland \& Linster, 1999), which is in accordance with other optical recording studies (Friedrich \& Korsching, 1997; Fuss \& Korsching, 2001; Wachowiak \& Cohen, 2001; Wachowiak et al., 2002).

\section{Effects of odour concentration on inhibited glomeruli}

In the PN signals odours evoke both excitation and inhibition (Sachse \& Galizia, 2002). Inhibitory responses are caused by inhibitory local interneurons, thus they are only visible in the output responses, not in the compound signals. These inhibitory PN responses were also dependent on the odour concentration. For example, the odourevoked calcium decrease of glomerulus 56 increased in negative amplitude and duration with increasing concentration of nonanol (Fig. 5A). We calculated the dose-response functions of inhibited glomeruli averaged over all measured animals; three of them are represented in Fig. 5B. All three glomeruli showed a significant increased inhibitory response with increasing odour concentration when comparing the glomerular responses to $10^{-5}$ with responses to $10^{-1}$ (two-tailed paired $t$-test: $P=0.036, P=0.044$ and $P=0.048$ for glomeruli 25, 29 and 49, respectively). Inhibition of glomeruli 25 and 29 saturated for the highest concentration. The maximum number of inhibited glomeruli appeared at odour concentrations of $10^{-5}$ and $10^{-4}$, and decreased with further increasing concentration (Fig. 5C). More olfactory receptor types become activated with increasing odour concentration (Vareschi, 1971; Firestein et al., 1993; Duchamp-Viret et al., 2000), which is also apparent in the responses of unspecific glomeruli in the compound signal at high concentrations (Fig. 3). Therefore this increase is likely to counterbalance the cross-glomerular inhibitory connections, and thus reducing the number of inhibited glomeruli on the output level of the AL at very high concentrations.
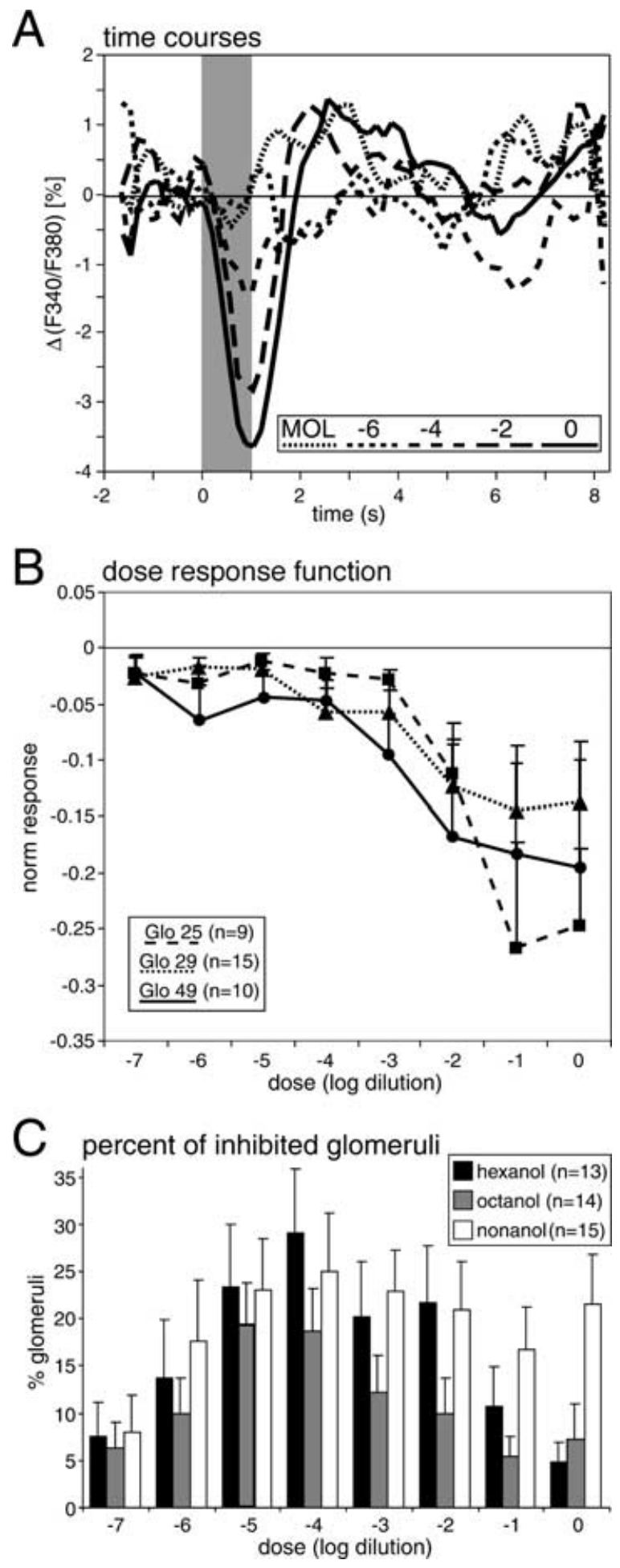

FIG. 5. In contrast to the compound signals, PNs showed inhibitory responses, which are also concentration-dependent. (A) Time traces of glomerulus 56 to nonanol stimulation. Stimulus is shown by the grey shading. The odour concentration is coded by the different line styles. As for the excitatory responses, increasing nonanol concentration increases the signal amplitude and duration. (B) Dose-response functions of three identified glomeruli to 8 different concentrations of nonanol averaged over all individuals (means and SEM). The responses have been normalized by setting the strongest inhibitory response in each animal for each odour at 1 and scaling the other responses accordingly. (C) Percentage of inhibited glomeruli to all three odours averaged over all animals measured. The number of inhibited glomeruli is maximal for intermediate concentrations. 


\section{Increasing odour concentration increases odour distinction}

In order to investigate how honeybees may recognize the same odour at different intensities, we analysed odour similarities across concentrations and asked whether odour-evoked activity patterns retain their odour-specificity. In a first approach we looked at a glomerular subset by representing the odour-evoked patterns in a three-dimensional space based on the response intensities of the three strongest and prominent glomeruli for the tested odours (glomeruli 28, 17 and 33 for hexanol, octanol and nonanol, respectively). Arranging the data in this way reveals that the compound responses (Fig. 6, Ai) above a concentration of $10^{-3}$ and the PN responses (Fig. 6, Aii) above $10^{-4}$ are clearly separable and are ranked from the centre to the periphery of the space with increasing concentration, whereas the activity patterns at lower concentrations are clustered in the centre. This clustering reflects the weaker overall activity elicited by low concentrations. We therefore visualized the excitation ratio as a vector between these three glomeruli for each odour at each concentration. Comparison of the compound activity patterns with the PN patterns shows that for all odours the different concentrations cluster together, while the odours are represented in three separate clusters on the PN level with exception of the very low concentrations. In comparison, the compound patterns do not clearly segregate. One reason for this difference is the strong contribution of glomerulus 17 to hexanol in the compound response, but not in the $\mathrm{PN}$ response (Figs 3, Bi, and $4 \mathrm{~A}$ and $\mathrm{B}$ ). We next analysed whether the odour representations become more distinct when all frequently imaged glomeruli $(n=23)$ are taken into account, again taking each glomerulus as an independent dimension in a multidimensional space. Using a principal component analysis (PCA; see Materials and methods) we visualized the relative positions of the odour responses in a reference system with reduced dimensionality. The PCA reveals that most of the variance (see figure legend) could be represented in a two-dimensional space, therefore only the first and second principal components are shown (Fig. 6B). Including all glomeruli separates the odour representations to a lesser extent than using only the three strongest glomeruli. The compound signals of hexanol were separated from octanol and nonanol only at the highest two concentrations. The activity patterns of octanol at higher concentrations appeared removed from nonanol for the third principal component (not shown). Nevertheless, when excluding the three dominant glomeruli (i.e. glomeruli 17, 28 and 33), the arrangement of the odour responses appeared almost unchanged, indicating that weakly activated glomeruli also contain considerable information about the olfactory stimulus. Interestingly, the multidimensional representations reflected the chemical similarity of the odours. Activity patterns evoked by octanol were arranged in between those of hexanol and nonanol, but closer to the latter. The 'gap' between hexanol and octanol is filled by glomerular responses to heptanol, as shown previously for the compound signal (Sachse et al., 1999).

As for electrophysiological recordings of frog OSNs (DuchampViret et al., 1990b), these analyses show that the odour representations become more distinct with stimulus intensity, as the further the odour is located from the centre, the longer its distance from the other odours. The continuity of OSN recruitment, which leads to the PNs becoming more and more active with increasing stimulus concentration, probably coincides with a more detailed description of the odours.

\section{Discussion}

In this study, we used calcium imaging in the honeybee to investigate the processing of odour quality and intensity by the AL network. To this end, we simultaneously measured input and output to the AL when stimulating with different odours at varying concentrations. A comparison of the two provides evidence that the primary olfactory signals are modulated and contrast-enhanced by the AL network to improve both concentration-invariance for odour quality detection and the sensitivity of the olfactory system.

\section{Methodological considerations}

Fura-dextran was locally applied to the lateral ACT in order to selectively stain uniglomerular PNs, which innervate the superficial glomeruli, whereas the calcium green-2AM was bath-applied to the whole AL to measure the OSNs.

The direct input from OSNs to PNs is mediated by nicotinic acetylcholine receptors (Bicker, 1999), which are calcium-permeable in cultured honeybee neurons (Goldberg et al., 1999). In locusts the calcium influx through these receptors contributes approximately $25 \%$ to the total calcium signal (Oertner et al., 1999). Thus the calcium measured in the PNs in this study may consist of both a component reflecting the input to the $\mathrm{PN}$ and a component proportional to the membrane potential, thus corresponding to the output from the PN. Therefore the calcium responses may overestimate the PN spike output to the mushroom bodies. However, studies combining electro-physiological and opto-physiological recordings of olfactory output neurons in rats (Charpak et al., 2001) and honeybees (Kimmerle \& Menzel, 2000) showed a high concordance between both techniques, suggesting that calcium responses overestimate the PN output only to a limited extent.

Calcium signals deriving from the two dyes could be separated optically. In control experiments when we applied only one staining protocol at a time, we observed no or only weak signals at the wavelength for the other, non-used dye (not shown). This is further confirmed by the different response properties of the two neuron populations. In contrast to the compound responses, the PN signals showed spontaneous activity (Fig. 1), a faster rising time and lasted only until the stimulus offset for intermediate odour concentrations. Furthermore, the compound differed from the PN responses in that signals could be observed in glomeruli which showed no calcium response at the PN level (e.g. glomerulus 39 to nonanol, Fig. 3).

In contrast to other studies (Firestein et al., 1993; Duchamp-Viret et al., 1999; Duchamp-Viret et al., 2000; Spors \& Grinvald, 2002) we could not observe any changes in latency of the odour responses with increasing odour concentration. However, our temporal resolution of $6 \mathrm{~Hz}$ was too low to detect changes in latency that were reported to be in the range of $80 \mathrm{~ms}$ (Spors \& Grinvald, 2002).

\footnotetext{
FIG. 6. Increasing odour concentration leads to activity patterns becoming less similar to each other. (A) The averaged compound (Ai) as well as PN activity patterns (Aii) to the three alcohols over eight concentrations are represented in a three-dimensional space defined by the normalized response intensity of the three strongest glomeruli (left; as shown in Fig. 4A and B) and their relative excitation ratios (right). Each activity pattern can be represented as a point in space or a vector with a specific length (response intensity) and direction (relative excitation). Transforming these vectors to unit length corrects for unequal response intensities (right). The colours indicate the odour, the numbers the odour concentration. Both representations reveal that the three odours are more clearly separated at the PN level than as compound patterns. (B) Principal component analyses (PCA) of the compound (Bi) and PN responses (Bii), performed on all measured individuals for all three odours. In the analyses we included either all 23 most frequently recognized glomeruli (left), all except the three glomeruli most sensitive to the used odour panel (i.e. 17,28 and 33, middle) or only these most sensitive glomeruli (corresponding to the data in (A), right). The projection in the two-dimensional plane accounts for most of the variance (comp. sig. 76\%, 82\% and 97\%; PN: 88\%, 88\% and 98\%, respectively). The best separation of the stimuli is obtained when only the three most sensitive glomeruli are considered in the analysis. In all cases the separation of odour-specific responses improved with increasing concentration.
} 


\section{Ai Compound signal}

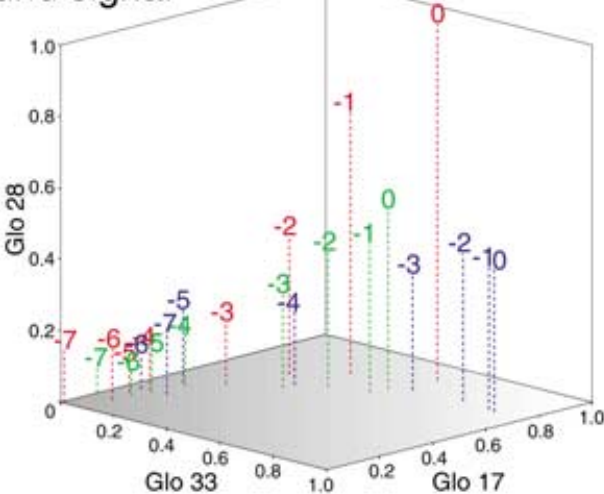

Aii PN signal

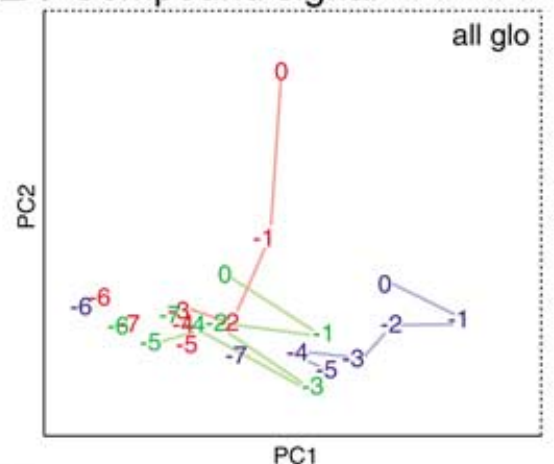

\section{Bii PN signal}

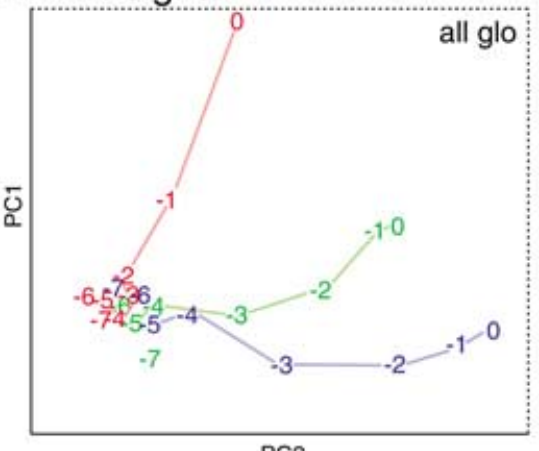

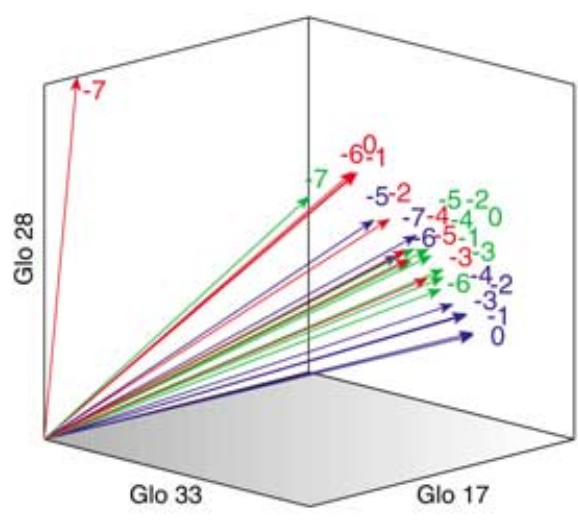

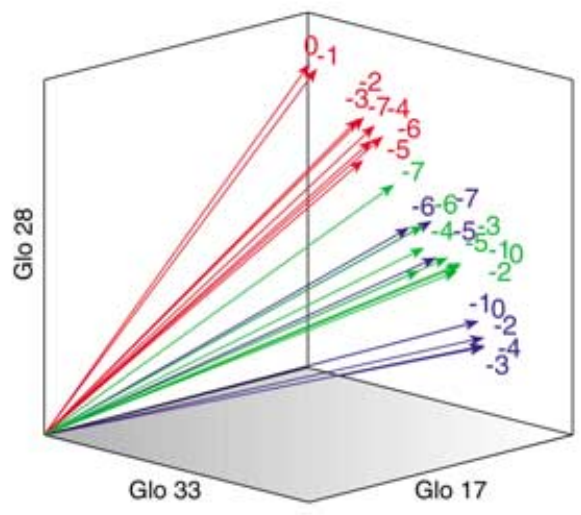

\section{Bi Compound signal \\ hexanol octanol nonanol}
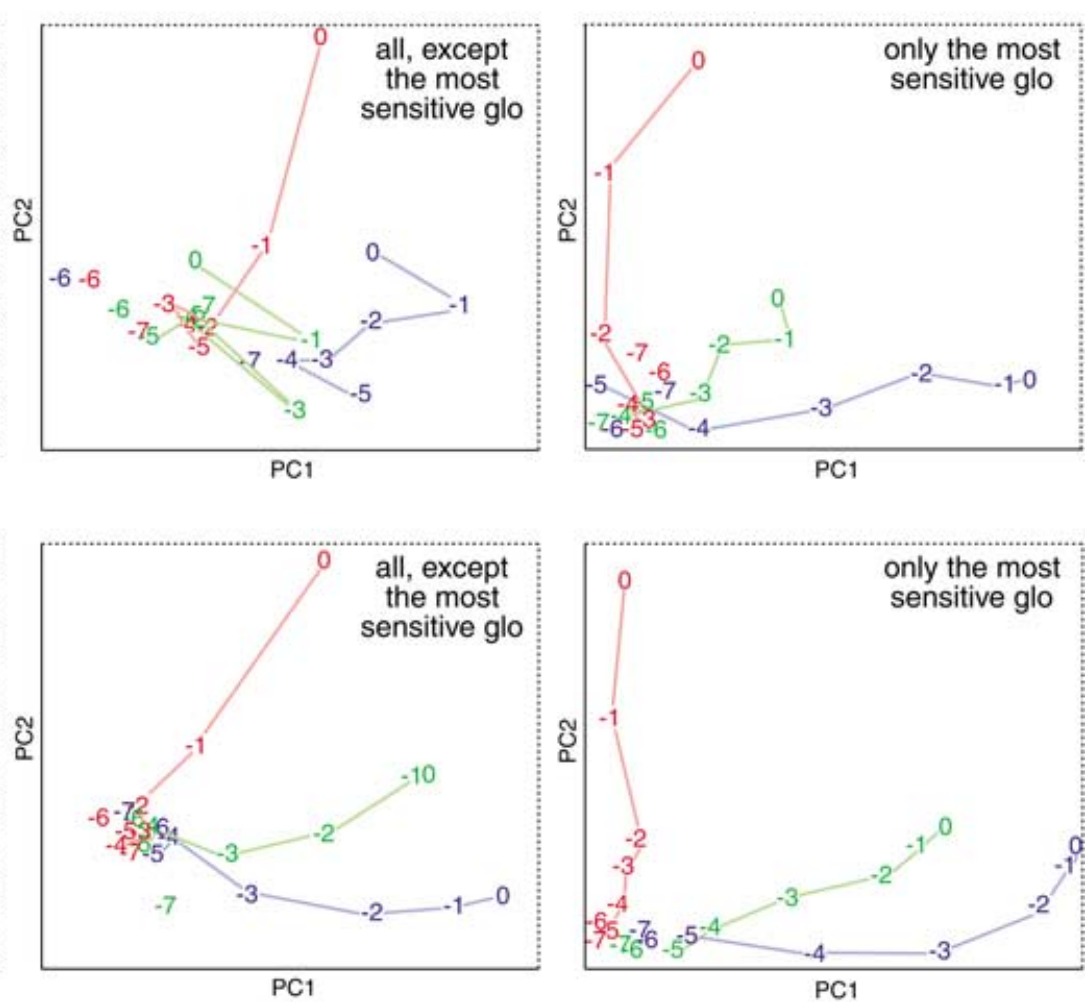


\section{The output odour representation is optimized by the AL network}

With increasing odour concentration, the receptive range of individual OSNs becomes broader (Vareschi, 1971; Akers \& Getz, 1993; Firestein et al., 1993; Duchamp-Viret et al., 2000; Ma \& Shepherd, 2000; de Bruyne et al., 2001) leading to a decrease of their odourspecificity. This broadening has to be corrected for the olfactory system to ensure reliable odour discrimination. Indeed, our results show that the AL network improves the afferent input by sharpening the glomerular response profiles and thus contrast-enhancing the representation of different odours. The dose-response functions of the less sensitive glomeruli were shifted towards higher concentrations, leading to a lower number of glomeruli showing an excitatory output compared to the input signal (Figs 2B and 3, Table 1). On the other hand, the sensitivity of glomeruli, which were the strongest at the highest odour concentration, was increased on the output level (e.g. glomerulus 28 for hexanol and 17 for octanol; Fig. 4C, Table 1), while the relative responses of a few others which dominated the activity patterns at low concentrations were reduced (e.g. glomerulus 17 for hexanol or nonanol). As a result, each odour elicited activity in only a few glomeruli even at very low concentrations. Our findings are in line with a comparative electrophysiological study of frog OSNs and second-order neuron sensitivities, which reported that the odour response thresholds within second-order neurons were significantly lower than thresholds in OSNs (Duchamp-Viret et al., 1989). This sensitivity improvement may be due to the strong convergence of OSNs onto mitral cells or PNs (Schild, 1988; Duchamp-Viret et al., 1989; Mori \& Shepherd, 1994; Laurent, 1999). The observed contrast-enhancement of glomerular responses is probably accomplished by inhibitory connections via LNs within the honeybee AL (Sachse \& Galizia, 2002). As a result of the increased OSN input with increasing concentrations, one would expect an amplification of inhibitory inputs to PNs, which should be visible in their glomerular responses. Indeed, glomeruli that were inhibited by a specific odour showed an increased inhibitory response with increasing concentration (Fig. 5A and B). However, we also observed that the total number of inhibited glomeruli decreased again at very high concentrations (Fig. 5C). These two opposing effects may be due to distinct types and strengths of inhibitory input to PNs. We previously proposed a putative glomerular connectivity model which implies two independent inhibitory networks (Sachse \& Galizia, 2002): an inhibitory network affecting an unspecific global gain control mechanism, and a separate inhibitory network that mediates glomerulus-specific inhibition to contrast-enhance responses of glomeruli with overlapping response profiles. Strongly and specifically inhibited glomeruli remain inhibited also at high odour concentrations, whereas glomeruli affected by the global unspecific inhibitory network might become activated with increased receptor neuron input and thus with rising odour intensity.

\section{Coding properties of odour intensity}

Foraging honeybees experience odours from the same flower at varying concentrations. Behavioural studies have shown that honeybees are capable of generalizing odour quality over a range of concentrations, which suggests that they would be unable to use odourant intensity to discriminate (Bhagavan \& Smith, 1997; Pelz et al., 1997). However, other studies have shown that bees can clearly discriminate two concentrations of the same odour when the odour intensities differ by a factor of 100 (Vareschi, 1971; Kramer, 1976; Getz \& Smith, 1991). This difference can even be less, if bees are trained in many learning trials (Ditzen \& Galizia, 2002). These findings imply that the olfactory system is able to extract both, the odour identity as well as its intensity. An often-stated requirement for odour coding is therefore that odour quality be perceived and possibly processed independently from odour intensity. As a corollary, odour quality information should be largely concentrationinvariant. Information about odour intensity may be encoded by the overall glomerular activity strength. This information may be processed by the pluriglomerular PNs, which innervate many glomeruli homogeneously and project directly to the lateral protocerebrum via the medio-lateral ACT (Fonta et al., 1993; Abel et al., 2001). A concentration-invariant code of odour quality could be realized by different conceivable mechanisms. One strategy could be a read-out of the glomerular order in terms of their relative response intensity. The sequences of active glomeruli in the honeybee AL both at the input and the output side were stable for 1-2 log units, confirming previous reports from honeybee optical imaging studies that reported near intensity-invariance over 2 log-units (Joerges et al., 1997; Sachse et al., 1999). However, some glomeruli had steeper response functions than others (Fig. 3, Table 1), leading to shifts in the sequence of the most-activated glomeruli and thus substantial changes in the relative activity patterns over a wider concentration range. These findings are in line with several imaging studies of vertebrates (Friedrich \& Korsching, 1997; Johnson \& Leon, 2000; Fuss \& Korsching, 2001; Wachowiak \& Cohen, 2001; Fried et al., 2002) and invertebrates (Sachse et al., 1999; Galizia et al., 2000), but in contrast to OSNs of turtles, whose glomerular response hierarchy changed only slightly over a concentration range of up to three log units (Wachowiak et al., 2002). It is unclear whether these shifts lead to a shift in perceived odour quality as would be predicted, if the sequence of glomerular activities was to encode odour quality. In that case, concentration-invariance would only be apparent for a small intensity range. Our present data suggest a better strategy for intensity-invariance in a manner similar to that used by the visual system, where three types of cones accomplish an intensity-invariant colour perception by means of colour-opponent encoding. The excitation ratio between the most responsive glomeruli could be the relevant read-out, which is shown for the three strongest glomeruli in Fig. 6A as the direction of the vectors. On the input level this ratio appears strongly concentration-dependent (Fig. 6, Ai), whereas the odour responses of the output neurons revealed an almost concentration-invariant excitation ratio over a concentration range of up to $4 \log$ units due to the glomerulus-specific and odour-specific contrast-enhancement (Fig. 6, Aii). The direction of the vectors is only weakly affected by the different steepness of glomerular response curves. At the same time, the representation of odour intensity is improved due to the continuous increase of the overall excitation in the PNs with increasing concentration. Thus, the AL network optimizes odour representations by separating odour quality information from intensity. Still, psychophysical experiments have to be performed to analyse whether the odour quality does indeed persist over the range of concentrations tested to verify the assumptions.

Interestingly, when only the three most sensitive glomeruli are considered, odours at low concentrations are more clearly separated (Fig. 6B). This indicates that the contrast-enhancement, which optimizes excitation ratios, is not perfect and could further be improved by higher processing centres, such as the lateral protocerebrum and/or the mushroom bodies, by selectively filtering only the strongest glomerular responses. This raises the question of what mechanism might account for such a selective read-out, if it exists. In locusts the spikes of coactivated PNs are often synchronized (Laurent et al., 1996; Wehr \& Laurent, 1996) by the distributed action of GABAergic LNs (MacLeod 
\& Laurent, 1996). It is conceivable that PNs from strong glomeruli may synchronize to a greater extent than those from weaker glomeruli and in this way form the only signal that is read by the mushroom body. In order to investigate this aspect it would be necessary to record from identified glomeruli with high temporal resolution, which is not possible with calcium-sensitive dyes but may be feasible with voltage-sensitive dyes or targeted electrodes.

Taken together, the results show that signal integration within the AL allows for a concentration-invariant coding of odour quality in the relative responses of glomeruli, creates a reliable representation of odour intensity in the overall AL activity, and improves odour-identification at low concentrations. Future experiments dedicated to higher order brain centres will help to describe the whole processing path of the olfactory code, ultimately ranging from the OSN level to the animal's behavioural response.

\section{Acknowledgements}

We thank Randolf Menzel for helpful discussions and support, Philipp Peele for critical comments on the manuscript and Beate Eisermann for excellent technical assistance. This work was supported by the Volkswagenstiftung (VW 1/75-399) and HFSP (RGY50/2001-B).

\section{Abbreviations}

ACT, antenno-cerebralis tract; AL, antennal lobe; LN, local interneuron; OSN, olfactory sensory neuron; PCA, principal component analysis; PN, projection neuron.

\section{References}

Abel, R., Rybak, J. \& Menzel, R. (2001) Structure and response patterns of olfactory interneurons in the honeybee, Apis mellifera. J. Comp. Neurol., 437, 363-383.

Akers, R.P. \& Getz, W.M. (1993) Response of olfactory receptor neurons in honeybees to odorants and their binary mixtures. J. Comp. Physiol. A, 173, 169-185.

Bhagavan, S. \& Smith, B.H. (1997) Olfactory conditioning in the honeybee Apis mellifera: Effects of odor intensity. Physiol. Behav., 61, 107-117.

Bicker, G. (1999) Histochemistry of classical neurotransmitters in antennal lobes and mushroom bodies of the honeybee. Micros. Res. Techn., 45, 174-183.

de Bruyne, M., Foster, K. \& Carlson, J.R. (2001) Odor coding in the Drosophila antenna. Neuron, 30, 537-552.

Charpak, S., Mertz, J., Beaurepaire, E., Moreaux, L. \& Delaney, K. (2001) Odor-evoked calcium signals in dendrites of rat mitral cells. Proc. Natl Acad. Sci., 98, 1230-1234.

Cleland, T.A. \& Linster, C. (1999) Concentration tuning mediated by spare receptor capacity in olfactory sensory neurons: a theoretical study. Neural Comput., 11, 1673-1690.

Ditzen, M. \& Galizia, C.G. (2002) Successfull odor identification in the honeybee Apis mellifera happens within an odor-independent time window. $X V$. Biennal Congress of ECRO (European Chemoreception Research Organisation), Erlangen, Germany, July 23-27, 2002. Abstracts, p. 39.

Duchamp-Viret, P., Chaput, M.A. \& Duchamp, A. (1999) Odor response properties of rat olfactory receptor neurons. Science, 284, 2171-2174.

Duchamp-Viret, P., Duchamp, A. \& Chaput, M.A. (2000) Peripheral odor coding in the rat and frog: Quality and intensity specification. J. Neurosci., 20, 2383-2390.

Duchamp-Viret, P., Duchamp, A. \& Sicard, G. (1990b) Olfactory discrimination over a wide cocentration range. Comparison of receptor cell and bulb neuron abilities. Brain Res., 517, 256-262.

Duchamp-Viret, P., Duchamp, A. \& Vigouroux, M. (1989) Amplifying role of convergence in olfactory system: a comparative study of receptor cell and second-order neuron sensitivities. J. Neurophysiol., 61, 1085-1094.

Duchamp-Viret, P., Duchamp, A. \& Vigouroux, M. (1990a) Temporal aspects of information processing in the first two stages of the frog olfactory system: influence of stimulus intensity. Chem. Senses, 15, 349-365.

Esslen, J. \& Kaissling, K.-E. (1976) Zahl und Verteilung antennaler Sensillen bei der Honigbiene (Apis mellifera L.). Zoomorphol., 83, 227-251.
Firestein, S., Picco, C. \& Menini, A. (1993) The relation between stimulus and response in olfactory receptor cells of the tiger salamander. J. Physiol. (Lond.), 468, 1-10.

Flanagan, D. \& Mercer, A.R. (1989) An atlas and 3-D reconstruction of the antennal lobe in the worker honey bee, Apis mellifera L. (Hymenoptera: Apidae). Int. J. Insect Morph. Embryol., 18, 145-159.

Fonta, C., Sun, X.J. \& Masson, C. (1993) Morphology and spatial distribution of bee antennal lobe interneurons responsive to odours. Chem. Senses, 18, 101-119.

Fried, H.U., Fuss, S.H. \& Korsching, S.I. (2002) Selective imaging of presynaptic activity in the mouse olfactory bulb shows concentration and structure dependence of odor responses in identified glomeruli. Proc. Natl Acad. Sci., 99, 3222-3227.

Friedrich, R.W. \& Korsching, S.I. (1997) Combinatorial and chemotopic odorant coding in the zebrafish olfactory bulb visualized by optical imaging. Neuron, 18, 737-752.

Fuss, S.H. \& Korsching, S.I. (2001) Odorant feature detection: activity mapping of stucture response relationships in the zebrafish olfactory bulb. J. Neurosci., 21, 8396-8407.

Galizia, C.G., McIllwrath, S.L. \& Menzel, R. (1999a) A digital three-dimensional atlas of the honeybee antennal lobe based on optical sections acquired by confocal microscopy. Cell Tissue Res., 295, 383-394.

Galizia, C.G., Nägler, K., Hölldobler, B. \& Menzel, R. (1998) Odour coding is bilaterally symmetrical in the antennal lobe of honeybees (Apis mellifera). Eur. J. Neurosci., 10, 2964-2974.

Galizia, C.G., Sachse, S. \& Mustaparta, H. (2000) Calcium responses to pheromones and plant odours in the antennal lobe of the male and female moth Heliothis virescens. J. Comp. Physiol. A, 186, 1049-1063.

Galizia, C.G., Sachse, S., Rappert, A. \& Menzel, R. (1999b) The glomerular code for odor representation is species specific in the honeybee Apis mellifera. Nature Neurosci., 2, 473-478.

Gao, Q., Yuan, B. \& Chess, A. (2000) Convergent projections of Drosophila olfactory neurons to specific glomeruli in the antennal lobe. Nature Neurosci., 3, 780-785.

Getz, W.M. \& Smith, K.B. (1991) Olfactory perception in honeybees: Concatenated and mixed odorant stimuli, concentration, and exposure effects. J. Comp. Physiol. A, 169, 215-230.

Goldberg, F., Grünewald, B., Rosenboom, H. \& Menzel, R. (1999) Nicotinic acetylcholine currents of cultured Kenyon cells from the mushroom bodies of the honeybee Apis mellifera. J. Physiol. (Lond.), 514, 759-768.

Hammer, M. (1997) The neural basis of associative reward learning in honeybees. Trends Neurosci., 20, 245-252.

Hildebrand, J.G. \& Shepherd, G.M. (1997) Mechanisms of olfactory discrimination: converging evidence for common principles across phyla. Anпи. Rev. Neurosci., 20, 595-631.

Joerges, J., Küttner, A., Galizia, C.G. \& Menzel, R. (1997) Representation of odours and odour mixtures visualized in the honeybee brain. Nature, 387, $285-288$.

Johnson, B.A. \& Leon, M. (2000) Modular representation of odorants in the glomerular layer of the rat olfactory bulb and the effects of stimulus concentration. J. Comp. Neurol., 422, 496-509.

Kimmerle, B. \& Menzel, R. (2000) Spatial and temporal processing of odor information in single antennal lobe neurons of the honeybee. Eur. $J$. Neurosci., 12, 498.

Kramer, E. (1976) The orientation of walking honeybees in odour fields with small concentration gradients. Physiol. Entomol., 1, 27-37.

Laurent, G. (1999) A system perspective on early olfactory coding. Science, 286, 723-728.

Laurent, G., Wehr, M. \& Davidowitz, H. (1996) Temporal representation of odors in an olfactory network. J. Neurosci., 16, 3837-3747.

Ma, M. \& Shepherd, G.M. (2000) Functional mosaic organization of mouse olfactory receptor neurons. Proc. Natl Acad. Sci., 97, 12869-12874.

MacLeod, K. \& Laurent, G. (1996) Distinct mechanisms for synchronization and temporal patterning of odor-encoding neural assemblies. Science, 274, 976-979.

Malnic, B., Hirono, J., Sato, T. \& Buck, L.B. (1999) Combinatorial receptor codes for odors. Cell, 96, 713-723.

Meister, M. \& Bonhoeffer, T. (2001) Tuning and topography in an odor map on the rat olfactory bulb. J. Neurosci., 21, 1351-1360.

Mombaerts, P., Wang, F., Dulac, C., Chao, S.K., Nemes, A., Mendelsohn, M., Edmondson, J. \& Axel, R. (1996) Visualizing an olfactory sensory map. Cell, 87, 675-686.

Mori, K. \& Shepherd, G.M. (1994) Emerging principles of molecular signal processing by mitral/tufted cells in the olfactory bulb. Sem. Cell. Biol., 5, $65-74$. 
Oertner, T.G., Single, S. \& Borst, A. (1999) Separation of voltage- and ligandgated calcium influx in locust neurons by optical imaging. Neurosci. Lett., 274, 95-98.

Pelz, C., Gerber, B. \& Menzel, R. (1997) Odorant intensity as a determinant for olfactory conditioning in honeybees: roles in discrimination, overshadowing and memory consolidation. J. Exp. Biol., 200, 837-847.

Ressler, K.J., Sullivan, K.J. \& Buck, L.B. (1994) Information coding in the olfactory system: evidence for a stereotyped and highly organized epitope map in the olfactory bulb. Cell, 79, 1245-1255.

Rubin, B.D. \& Katz, L.C. (1999) Optical imaging of odorant representations in the mammalian olfactory bulb. Neuron, $\mathbf{2 3}, 499-511$.

Sachse, S. \& Galizia, C.G. (2002) Role of inhibition for temporal and spatial odor representation in olfactory output neurons: a calcium imaging study. $J$. Neurophysiol., 87, 1106-1117.

Sachse, S., Rappert, A. \& Galizia, C.G. (1999) The spatial representation of chemical structures in the antennal lobes of honeybees: steps towards the olfactory code. Eur. J. Neurosci., 11, 3970-3982.

Sato, T., Hirono, J., Tonoike, M. \& Takebayashi, M. (1994) Tuning specificities to aliphatic odorants in mouse olfactory receptor neurons and their local distribution. J. Neurophysiol., 72, 2980-2989.

Schild, D. (1988) Principles of odor coding and a neural network for odor discrimination. Biophys. J., 54, 1001-1011.

Sicard, G. \& Holley, A. (1984) Receptor cell responses to odorants: similarities and differences among odorants. Brain Res., 292, 283-296.

Spors, H. \& Grinvald, A. (2002) Spatio-temporal dynamics of odor representations in the mammalian olfactory bulb. Neuron, 34, 301-315.
Trotier, D. (1994) Intensity coding in olfactory receptor cells. Sem. Cell. Biol., $\mathbf{5}, 47-54$.

Uchida, N., Takahashi, Y.K., Tanifuji, M. \& Mori, K. (2000) Odor maps in the mammalian olfactory bulb: domain organization and odorant structural features. Nature Neurosci., 3, 1035-1043.

Vareschi, E. (1971) Duftunterscheidung bei der Honigbiene - EinzelzellAbleitungen und Verhaltensreaktionen. Z. Vergl. Physiol., 75, 143173.

Vassar, R., Chao, S.K., Sitchran, R., Nuñez, J.M., Vosshall, L.B. \& Axel, R. (1994) Topographic organization of sensory projections to the olfactory bulb. Cell, 79, 981-991.

Vosshall, L.B., Wong, A.M. \& Axel, R. (2000) An olfactory sensory map in the fly brain. Cell, 102, 147-159.

Wachowiak, M. \& Cohen, L.B. (2001) Representation of odorants by receptor neuron input to the mouse olfactory bulb. Neuron, 32, 723-735.

Wachowiak, M., Cohen, L.B. \& Zochowski, M.R. (2002) Distributed and concentration-invariant spatial representations of odorants by receptor neuron input to the turtle olfactory bulb. J. Neurophysiol., 87, 10351045.

Wehr, M. \& Laurent, G. (1996) Odour encoding by temporal sequences of firing in oscillating neural assemblies. Nature, 384, 162-166.

Witthöft, W. (1967) Absolute Anzahl und Verteilung der Zellen im Hirn der Honigbiene. Z. Morph. Tiere, 61, 160-184.

Yokoi, M., Mori, K. \& Nakanishi, S. (1995) Refinement of odor molecule tuning by dendrodendritic synaptic inhibition in the olfactory bulb. Proc. Natl Acad. Sci., 92, 3371-3375. 